\title{
ERRORES EN LA IDENTIFICACIÓN DE NÚMEROS REALES COMETIDOS POR INGRESANTES UNIVERSITARIOS
}

Mendoza, M.E. ${ }^{(*) 1}$, Caputo, L.N..$^{(*)}$, Bordón, Paula D. ${ }^{(*)}$, Porcel, Eduardo A. ${ }^{(*) 4}$

Palabras claves: Errores, Subconjuntos de números reales, Identificación, Ingresantes universitarios.

Resumen: En este trabajo se realiza un análisis estadístico descriptivo de los errores cometidos por ingresantes a las carreras Ingeniería Eléctrica, Ingeniería en Agrimensura, Ingeniería en Electrónica, Licenciatura en Ciencias Físicas y Licenciatura en Ciencias Químicas al identificar números reales como tales o como elementos de los conjuntos de números naturales, enteros, racionales e irracionales. Se pudo observar elevados porcentajes de errores que dan cuenta de las dificultades de los estudiantes para identificar al 0 como un número racional, entero y no natural, como racionales a aquellos números racionales con infinitas cifras periódicas y como número complejo no real a la raíz cuadrada de un número real negativo. Puede concluirse que los errores cometidos están vinculados a concepciones alternativas de los estudiantes las cuales provienen de hábitos escolares habituales a lo largo de la escolaridad preuniversitaria.

\section{Introducción}

Desde hace algunos años es un hecho conocido y aceptado por los docentes y autoridades de las Instituciones de Educación Superior que los elevados niveles de fracaso estudiantil en los primeros años de estudios de las carreras de grado del Nivel Superior están íntimamente ligado a la formación de los jóvenes en los niveles primario y secundario.

Esta problemática se acentúa aún más en el caso de estudiantes universitarios de carreras técnicas o relacionadas con las ciencias exactas y naturales.

En efecto, Caputo et al. [1] sostiene que "las cátedras universitarias de matemática de primer año reciben el impacto de serias deficiencias en la preparación básica que en esta disciplina tienen los ingresantes a las carreras de Nivel Superior" (p.57). Por su parte Nabarro et al. [2] completan esta idea

$\left(^{*}\right)$ Facultad de Ciencias Exactas y Naturales y Agrimensura. Universidad Nacional del Nordeste. Avda. Libertad 5450. 3400. Corrientes.

1- Profesora de Matemática; mat.mendoza1812@gmail.com

2- Especialista en Docencia Universitaria; proflcaputo@gmail.com

3- Profesora de Matemática; paulabordon85@gmail.com

4- Magíster en Estadística Aplicada; porcelfel@arnet.com.ar 
al afirmar que los profesores de dichas cátedras reciben "...alumnos del nivel secundario con escasos saberes disciplinares, hábitos de estudio memorístico, actitud pasiva en las clases, desmotivados, etc..." (p.183)

Para paliar el problema de las deficiencias de formación de sus ingresantes, la mayoría de las universidades institucionalizó cursos de nivelación o de admisión, previos al inicio de los estudios de grado. Entre los cursos de admisión más conocidos merecen mencionarse el Ciclo Básico Común (CBC) de la Universidad Nacional de Buenos Aires (UBA) y el Seminario Universitario de la Universidad Tecnológica Nacional (UTN).

La Universidad Nacional del Nordeste (UNNE), no tiene una política unificada respecto al ingreso de estudiantes a la Institución, sino que dicha política difiere según la unidad académica. La Facultad de Ciencias Exactas y Naturales y Agrimensura (FACENA) ha mantenido desde 1985 hasta la fecha una política de ingreso irrestricto. Sin embargo, en el período 2001-2013, se dictó — en el mes de febrero de cada año- un curso de nivelación consistente en tres módulos: Introducción a la vida universitaria, Matemática y un $3^{\circ}$ referido a la disciplina específica vinculada a la carrera elegida (Física, Química, Biología, Algebra o Informática). Si bien al final del curso se implementaba una prueba a fin de evaluar los aprendizajes resultantes de las actividades de enseñanza realizadas en el curso, la aprobación de dicha evaluación no era requisito necesario para el cursado de las asignaturas de la carrera elegida.
Al inicio de estos cursos, el grupo de Matemática Aplicada a la Investigación Educativa en tres oportunidades (2001, 2009 y 2013), suministró a los ingresantes un test de conocimientos matemáticos previos y, posteriormente, analizó en profundidad y mediante distintas metodologías los errores cometidos por los estudiantes evaluados. Los resultados obtenidos, permitieron concluir que el conjunto de números reales, así como también las definiciones y propiedades de las operaciones que usualmente se definen en dicho conjunto, es uno de los contenidos en el que se presentan las mayores deficiencias de conocimientos de los ingresantes. Por ello, al inicio del ciclo lectivo 2017, dicho grupo de investigación suministró a los ingresantes de las carreras Ingeniería Eléctrica, Ingeniería en Agrimensura, Ingeniería en Electrónica, Licenciatura en Ciencias Físicas y Licenciatura en Ciencias Químicas, una evaluación diagnóstica similar a las antes mencionadas, pero con el objetivo de explorar los saberes de estos alumnos referidos sólo al conjunto de números reales. El test consistió en tres ejercicios, en los cuales se solicitó:

1) Identificar a:

$\frac{15}{3}, 3.75, \frac{22}{7}, 0, \frac{\sqrt{5}}{2}, \frac{-\sqrt{9}}{2}, \sqrt{6},-\sqrt{16}, \sqrt{-16}$ y $3 . \hat{9}$ como números reales y/o como elementos de alguno de sus subconjuntos $\mathbb{N}, \mathbb{Z}, \mathbb{Q}$ y $\mathbb{R}-\mathbb{Q}$.

2) Representar en la recta real, 5 de los números dados en 1 .

3) Determinar el valor de verdad de 10 proposiciones referidas a las operaciones con números reales y a sus propiedades y justificar la respuesta. 
En este trabajo se realizó un análisis descriptivo de las respuestas al ítem 1 del test, detectando los errores más frecuentes en que incurren los alumnos, y se establecieron posibles causas que los llevan a co- $^{-}$ meterlos.

\section{Marco referencial y antecedentes}

Los errores en el aprendizaje han sido objeto de estudio de numerosos investigadores, principalmente en el campo de la Educación Matemática. "En la actualidad el error es considerado parte inseparable del proceso de aprendizaje. Los investigadores en educación matemática sugieren diagnosticar y tratar seriamente los errores de los alumnos, discutir con ellos sus concepciones erróneas, y presentarles luego situaciones matemáticas que les permitan reajustar sus ideas." (p.2) [3]

Antes de ahondar en la clasificación y causas asociadas a su producción, diremos que "Hablamos de error cuando el alumno realiza una práctica (acción, argumentación, etc.) que no es válida desde el punto de vista de la institución matemática escolar.”(p.69) [4].

Por su parte, Abrate et al. [5] afirman que, al revisar numerosos trabajos de análisis de errores se puede concluir que éstos “... se reiteran en los distintos años y ciclos que conforman el sistema educativo, los que resultan ser básicamente los mismos para cada contenido de la currícula."(p.12 y 13)

Cabe mencionar que la resolución del ejercicio analizado implica poner en juego tres habilidades que Delgado Rubí [6] considera fundamentales para la construcción del conocimiento matemático: interpretar (consiste en dotar de significado a los objetos matemáticos en función de ellos mismos o del contexto en que se presenten), identificar (determinar si un objeto matemático pertenece a una determinada clase de objetos que poseen ciertas características distintivas) y definir (determinar mediante una proposición las condiciones necesarias y suficientes de un objeto matemático).

Por su parte, Abrate et al. [5] tratan de explicar los errores de los estudiantes mediante dificultades de aprendizaje vinculadas a la complejidad de los objetos matemáticos, a los procesos del pensamiento matemático, a los procesos de enseñanza, a los procesos cognitivos de los alumnos y a sus actitudes afectivas y emocionales con respecto a esta ciencia. Por su parte, Astolfi [7] al tratar de vincular los errores que los alumnos cometen en una práctica matemática, hace referencia a "errores resultado de los hábitos escolares o de una mala interpretación de las expectativas", "errores ligados a las operaciones intelectuales implicadas" y "errores vinculados a las concepciones alternativas de los alumnos", lo cual en cierta manera coincide con lo señalado por Abrate et al.[5].

Al respecto, cabe mencionar que al analizar un ejercicio similar incluido en la prueba de diagnóstico de 2001, Porcel y Ramírez Arballo [8], concluyen que entre el 35 y $60 \%$ de los sujetos evaluados no reconocen las relaciones de inclusión que existen, que una gran mayoría no reconoce a los naturales como número racionales y que, en general, asocian los términos número 
racional con fracción, e irracional con raíz cuadrada. En cambio Caputo et al. [9], [10] dan cuenta de que los alumnos ingresantes a FACENA en 2009 y 2013, respectivamente, demuestran limitaciones en la definición restringida de radicación en $\mathrm{R}$ al no identificar, la gran mayoría, correctamente a $\sqrt{2},-\sqrt{49}$ y $\sqrt{(}(-1)$

\section{Discusión de resultados}

Cabe destacar que la muestra de alumnos con que se trabajó está constituida por los 253 estudiantes que asistieron el 24 de febrero de 2017 a la primera clase práctica de la asignatura Algebra y Geometría Analítica, la cual corresponde al primer cuatrimestre del primer año de estudios de las currículas de todas las carreras mencionadas.

En principio, ningún alumno respondió correctamente los 50 subítems incluidos en el ejercicio 1.

Comenzando por la identificación del 0 , puede observarse la dificultad de reconocerlo como número racional debido a la imposibilidad de verlo como el cociente de dos números enteros donde el numerador es múltiplo del denominador. Esta afirmación confirma lo dicho por Abrate et al. al analizar las respuestas de una prueba de conocimientos previos suministrada a ingresantes a Ciencias Económicas de la Universidad Nacional de Villa María: que la mayoría de los alumnos no pudieron “... dar un resultado final para el ejercicio que los conducía a 0/4."[5]. Por último, quizás por haber definido a los números naturales como cardinales de conjuntos finitos, alrededor del 25\% de los alumnos identifican al
0 como natural, pero sí el $82 \%$ lo identifica como entero.

En la Figura 1 puede observarse que los alumnos identificaron mayoritariamente $(80 \%)$ a $\frac{15}{3}$ como un número racional y real, aunque sólo un poco menos de la mitad reconoció que es un número natural y, en consecuencia, entero.

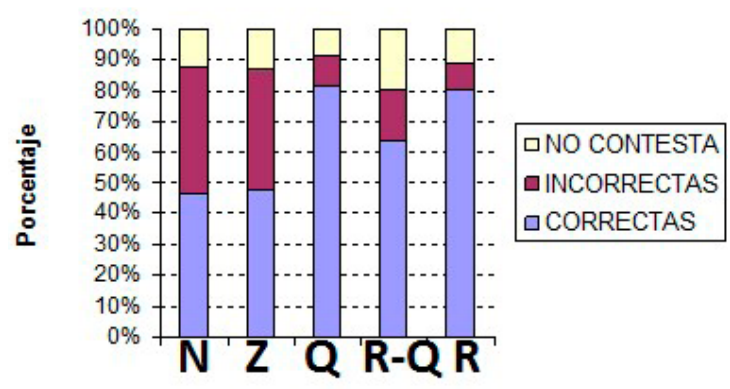

Figura 1: Identificación de $\frac{15}{3}$

Así pues, podemos observar que, una vez más, aparece aquí la asociación de número racional con fracción visto en [8], y parecería que el 80\% de los ingresantes reconocen a Q como un subconjunto de R. Sin embargo, estos niveles de respuestas correctas disminuyen casi en 10 puntos porcentuales en la identificación de $\frac{22}{7} \quad$ (Figura 2).

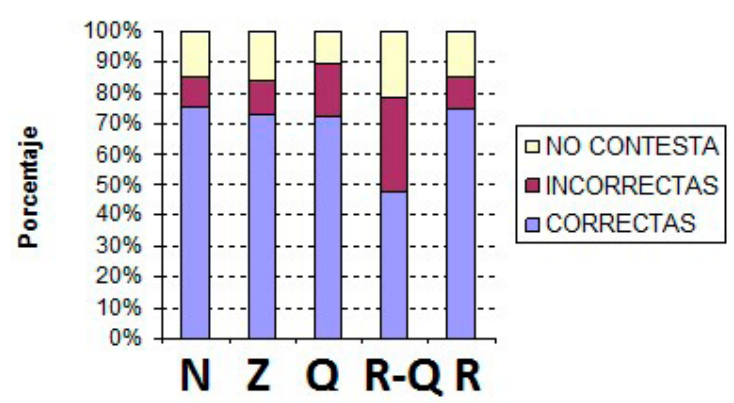

Figura 2: Identificación de $\frac{22}{7}$

Esta diferencia puede estar relacionada con el hecho de que al realizar el cociente de 22 por 7 mediante una calculadora se ob- 
tiene un número decimal de infinitas cifras periódicas; además de que dicho período es 142857 , la calculadora (por la limitación del número de cifras del visor) redondea la última cifra, consignando al último período como 143 , lo cual induce al $31 \%$ de los alumnos a pensar que se trata de un número irracional. Al respecto, Tabare [11] afirma que inducidos por prácticas escolares habituales, los alumnos suponen que, si las cifras del cociente cubren todo el visor de la calculadora, el mismo posee infinitas cifras decimales por lo cual “...buscarán reconocer el período, pero si no pueden observarlo podrían concluir que la expresión decimal posee infinitas cifras decimales no periódicas, es decir, asociar fracciones a números irracionales." Algo similar sucede con la identificación de 3. 9.: aunque más del 70\% lo reconoce como a un número real, sólo el $50 \%$ afirma que es racional y casi un $40 \%$ que no lo es. (Figura 3).

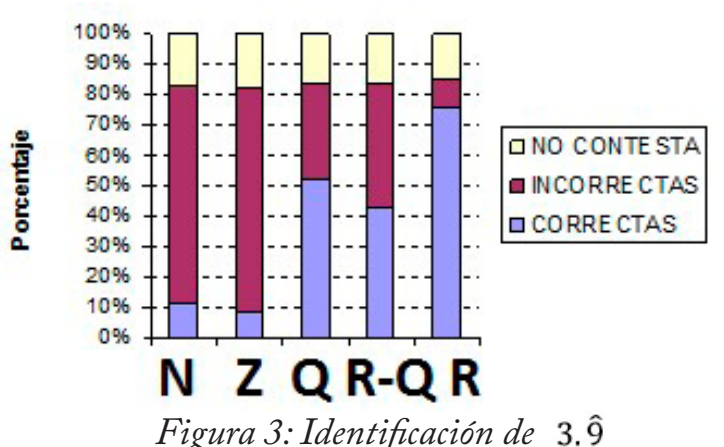

Nótese que Abrate et al. [5] afirman que al solicitarles la expresión fraccionaria de $2 . \hat{3}$, el $52 \%$ de los alumnos ingresantes a carreras de Ciencias Económicas de la Universidad Nacional de Villa María, aseguró que la misma es $\frac{23}{9}$, lo cual demuestra que, al tener un conocimiento memorístico respec- to al algoritmo que permite transformar en fracción a esta clase de expresiones decimales, no pueden usarlo correctamente cuando les es necesario. En la prueba analizada esto, sumado al hecho de que el período era 9, hizo que sólo un $11 \%$ lo identificara como natural y menos del 10\% como entero.

La confusión que genera en los estudiantes las definiciones de número racional e irracional, queda perfectamente demostrada al estudiar los porcentajes de respuestas respecto a 3.75: el 70\% lo identifica como real y racional no entero (y en consecuencia, no natural) y sólo un $55 \%$ dice que no es un número irracional. Así pues, puede decirse que hay un porcentaje de alumnos de alrededor del $76 \%$ que no tienen claro que Qy R- Q son disjuntos, porque todos ellos -en al menos un caso- respondieron que el número dado pertenecía a la intersección de ambos conjuntos.

En cuanto a la identificación de los números irracionales, un $50 \%$ y un $55 \%$ reconoce a $\sqrt{6} \mathrm{y} \frac{\sqrt{5}}{2}$ respectivamente como irracionales. En cambio, entre el 35 y el $42 \%$ reconocen como tales a $\frac{-\sqrt{9}}{2},-\sqrt{16} y \sqrt{-16}$ a pesar de que no lo son. Ėstos últimos porcentajes parecen confirmar lo observado por Porcel et al. de que los alumnos asocian los conceptos de número irracional con el de raíz cuadrada [8].

Cabe señalar que alrededor del $40 \%$ de estudiantes afirma que $\sqrt{6} \mathrm{y} \frac{\sqrt{5}}{2}$ son números racionales, en el primer caso la afirmación parece inducida por una confusión entre los conceptos de raíz cuadrada y el duplo de un número natural, el segundo, en cambio, podría explicarse mediante la concepción de 
que todo cociente es un número racional, coincidiendo nuevamente con los resultados de Porcel et al. [8] mencionados anteriormente. Esto último se comprueba con el hecho de que el $55 \%$ reconoce $\frac{-\sqrt{9}}{2}$ como elemento de $\mathbb{Q}$.

Por último, sólo un $40 \%$ reconoce que $\sqrt{-16}$ no es un número real, lo cual estaría indicando que para estos alumnos la definición de radicación en $\mathbb{R}$ constituye un obstáculo en el reconocimiento de elementos de los distintos conjuntos numéricos, coincidiendo con lo observado por Caputo et al. [9], [10].

\section{Conclusiones}

De los resultados obtenidos, puede concluirse que los errores más frecuentes se relacionan con concepciones alternativas de los alumnos que consisten principalmente en identificar radicandos con números irracionales y cocientes con números racionales. Éstas están vinculadas a las prácticas escolares habituales que limitan, generalmente, el trabajo en $\mathbb{Q}$ a la aritmética de fracciones y en $\mathbb{R}-\mathbb{Q}$, a la "racionalización" de denominadores, donde éstos incluyen al menos la raíz cuadrada de un número natural que no es un cuadrado perfecto.

Esta simplificación que hemos señalado es lo que obstaculiza que el alumno reconozca que estos dos conjuntos son disjuntos.

Un análisis generalizado de los resultados obtenidos permite concluir que la mayoría de los estudiantes tiene dificultades de identificar la existencia de las inclusiones: $\mathbb{N} \subset \mathbb{Z} \subset \mathbb{Q} \subset \mathbb{R}$, lo cual evidentemente le dificulta identificar a los números reales y posteriormente operar con ellos.

\section{REFERENCIAS}

[1] Caputo, L.; Porcel, E. Ibáñez, M.I.; Tirado, G.; Aliendro, E. (2003). Deficiencias en la formación matemática de los ingresantes universitarios. Libro de Resúmenes de la III Conferencia Argentina de Educación Matemática. SOAREM y Universidad Nacional de Salta. Salta.

[2] Nabarro, S.; Ibarra, E.; Cejas, C.; Ger, S. (2017). Las prácticas educativas y el desarrollo de competencias básicas en las carreras de la Facultad de Ciencias Forestales. Libro de Actas del XII Encuentro Internacional de Educación Matemática en carreras de Ingeniería,pp
183 - 189. Santiago del Estero: U.N. de Santiago del Estero. ISBN 978-987-720-151-2. [3] Del Puerto, S.; Minnaard, C.; Seminara, S. (2006) Análisis de los errores: una valiosa fuente de información acerca del aprendizaje de las $M a-$ temáticas. Revista Iberoamericana de Educación de la O.E.I. Volumen 38, Número Especial 38/4. pp 1-12. http://rieoei.org/1285. $\mathrm{htm}$. Accedido el 15 de septiembre de 2017.

[4] Godino, J.; Batanero C. y Font V. (2003). Fundamentos de la enseñanza y aprendizaje de la Matemática para maestros. Universidad de Granada. Accedido el 15 
de septiembre de 2017. Accedido el 15 de septiembre de 2017. http://www. ugr.es/local/jgdino/edumat-maestros/

[5] Abrate, R.; Pochulu, M.; Vargas, J. (2006). Errores y dificultades en Matemática. Análisis de causa y sugerencias de trabajo. 198 páginas. Universidad Nacional de Villa María. CABA. ISBN-10: 987-982929-8; ISBN-13: 978-987-98292-9-5.

[6] Delgado Rubí, J.R. (1995). Un sistema de habilidades para la enseñanza de la Matemática. Memorias del IX Reunión Centroamericana y de Caribe sobre la formación de Profesores e Investigación en Matemática Educativa. La Habana. Cuba. [7]Astolfi,J.P.El“error”, unmedioparaenseñar.Editorial DÍADA. 100 páginas. Sevilla. España.

[8] Porcel, E.; Ramírez Arballo, M.G. (2003). Determinación $y$ análisis de las principales deficiencias en la identificación de números pertenecientes a los distintos conjuntos numéricos: $\mathbb{N}, \mathbb{Z}, \mathbb{Q}, I$ ○ $\mathbb{R}$, en alumnos ingresantes a FACENA en 2001. Memorias del $\mathrm{V}$ Simposio de Educación $\mathrm{Ma}$ temática. Chivilcoy (Buenos Aires). Argentina. ISBN No $987-20239-1-3$.
[9] Caputo, L.N.; Porcel, E.A.; Romero, J.L. (2016). Explorando relaciones conceptuales sobre conjuntos numéricos de ingresantes universitarios con Análisis Estadístico Implicativo. Actas del 2o Congreso Internacional de enseñanza de las Ciencias y la Matemática. Tandil (Buenos Aires). Argentina. ISBN 978 - $950-658$ - $396-5$. [10] Caputo, L.N.; Jorge, M.J.; Espinoza, R.F.; Porcel, E.A.; Romero, J.L. (2016). Análisis Estadistico Implicativo de los conocimientos previos sobre números reales de ingresantes a la Universidad. Cadernos Do IME - Série Estatística. Volumen 41. pp 30 - 44. Universidade do Estado do Rio de Janeiro. Río de Janeiro. Brasil. ISSN impresso 1413 - 9022. ISSN on-line 2317 - 4535. [11] Tabare, M. (2014). Posibles errores en el aprendizaje de números reales a raiz del tratamiento didáctico propuesto en un texto del nivel secundario. Memoria de la Especialización en Didáctica de las ciencias con orientación en Matemática, dirigida por la Dra. Mabel Rodríguez. Universidad Nacional de General Sarmiento. 75 páginas. Los Polvorines (Buenos Aires). Argentina. 